\title{
On Integration of One Class of Systems of Lax-Type Equations
}

\author{
A.A. Lunyov \\ Institute of Applied Mathematics and Mechanics of the NAS of Ukraine \\ 74, R. Luxemburg Str., Donetsk 83114, Ukraine \\ E-mail: A.A.Lunyov@gmail.com \\ E.V. Oliynyk \\ V.N. Karazin Kharkiv National University \\ 4, Svobody Sq., Kharkiv 61022, Ukraine \\ E-mail: elenaoliynik@gmail.com
}

Received February 12, 2013, revised May 15, 2014

A nonlinear system of Lax-type equations is studied. The system is the basis of the construction of triangular models for commutative systems of linear non-selfadjoint bounded operators. Some of its solutions for $n=4$ are described. In one of the cases, the general solution is explicitly expressed in terms of special (elliptic) functions.

Key words: triangular models, nonlinear differential equations, commutative systems of linear non-selfadjoint operators.

Mathematics Subject Classification 2010: 47A48, 47N20, 34G20.

\section{Introduction}

As is well known from $[1,5]$, an approach based on Lax's idea to write the initial nonlinear equation in the form $L^{\prime}(x)=i[L(x), A(x)]$, where $L, A$ are some differential operators, is the main method of integration of nonlinear equations. It is established that if the Lax pair $\{L, A\}$ is found for a nonlinear equation, then this equation can be 'integrated'.

In this paper, we study the system of equations

$$
\frac{d}{d x}\left(\sigma_{2}+\lambda \gamma(x)\right)=i\left[a(x), \sigma_{2}+\lambda \gamma(x)\right], \quad \gamma(0)=\gamma^{+}, \quad x \in[0, l], \quad \lambda \in \mathbb{C},
$$


which is equivalent to the special system of the Lax-type equations

$$
\left\{\begin{array}{l}
{[a(x), \gamma(x)]=0, \quad x \in[0, l],} \\
\gamma^{\prime}(x)=i\left[a(x), \sigma_{2}\right], \quad x \in[0, l], \\
\gamma(0)=\gamma^{+}
\end{array}\right.
$$

where $a(x)$ is a spectral matrix measure, $\gamma(x), \sigma_{2}, \gamma^{+}$are selfadjoint $n \times n$ matrices, and

$$
a(x) \geqslant 0, \quad \operatorname{tr} a(x) \equiv 1, \quad x \in[0, l] .
$$

System (1.2) appears in the construction of triangular models for commutative systems of non-selfadjoint bounded operators.

The purpose of this paper is to describe and study all the pairs of matrix functions $\{a(x), \gamma(x)\}$, the solutions of system (1.2) (when $n=4$ ), for the given selfadjoint $n \times n$ matrices $\gamma^{+}$and $\sigma_{2}$ such that

$$
\gamma(x) \in A C\left([0, l] ; C^{n \times n}\right), \quad a(x) \in L^{1}\left([0, l] ; C^{n \times n}\right),
$$

and (1.3) takes place.

In [7], general qualities of the solutions of system (1.2) for the case $n=3$ are obtained and the descriptions of all solutions of this system for different cases are given. The idea of paper [7] is used in this paper. This idea lies in the fact that in the case when $\gamma^{+}$(and so $\gamma(x)$ also) has a simple spectrum, $a(x)$ is a polynomial of $\gamma(x)$ of no higher degree than $n-1$ (with scalar coefficients depending on $x$ ).

When $n=4$ and the matrices $\sigma_{2}, \gamma^{+}$have a simple spectrum, the explicit form of the solution in terms of elliptic functions is obtained (see Theorem 2.2 and Corollary 2.3). In Example 2.4, the solutions expressed by trigonometric functions are found.

When studying cubic dependency of $a(x)$ from $\gamma(x)(n=4)$, the explicit form of the solution is also expressed in terms of special (elliptic) functions (see Theorem 3.2 and Corollary 3.3).

\section{Description of the Solutions of System (1.2)}

Proposition 2.1. Let

$$
\sigma_{2}=\operatorname{diag}\left(b_{1}, \ldots, b_{n}\right), \quad \gamma^{+}=\alpha_{1} \sigma_{2}+\alpha_{0} I+i C,
$$

where $\alpha_{1}, \alpha_{0} \in R$, the matrix $C=\left(c_{j k}\right)_{j, k=1}^{n}=-C^{*}$ and $c_{j j}=0$ when $j \in$ $\{1, \ldots, n\}$.

Further, let $\kappa_{0}, \kappa_{1}, \kappa_{2} \in L^{1}[0, l]$ be real functions. Then the pair $\{a(\cdot), \gamma(\cdot)\}$, where $a(x)=\kappa_{2}(x) \gamma(x)^{2}+\kappa_{1}(x) \gamma(x)+\kappa_{0}(x), x \in[0, l]$ and $\gamma(\cdot)=\left(\gamma_{j k}(\cdot)\right)_{j, k=1}^{n}$, 
is the solution of system (1.2) if and only if the equalities

$$
\begin{aligned}
\gamma_{j j}(x) & =\gamma_{j j}^{+}, \quad j \in\{1, \ldots, n\}, \\
\gamma_{j k}(x) & =i \mathrm{e}^{i\left(b_{j}-b_{k}\right)\left(K_{1}(x)+\left(\gamma_{j j}^{+}+\gamma_{k k}^{+}\right) K_{2}(x)\right)} y_{j k}(x), \quad j \neq k,
\end{aligned}
$$

hold as $x \in[0, l]$, where

$$
K_{j}(x):=\int_{0}^{x} \kappa_{j}(t) d t, \quad j \in\{1,2\},
$$

and the functions $y_{j k}(\cdot), j \neq k$, satisfy the system

$$
\left\{\begin{array}{l}
y_{j k}^{\prime}(x)=\left(b_{k}-b_{j}\right) \kappa_{2}(x) \sum_{\substack{s=1 \\
s \neq j, k}}^{n} y_{j s}(x) y_{s k}(x), \quad x \in[0, l], \quad j \neq k, \\
y_{k j}(x)=\overline{-y_{j k}(x)}, \quad x \in[0, l], \quad j \neq k, \\
y_{j k}(0)=c_{j k}, \quad j \neq k .
\end{array}\right.
$$

Besides, if $c_{j k} \in R, j \neq k$, every solution of system (2.5) is real.

P r o o f. Since $a(x)$ commutes with $\gamma(x)$, then system (1.2) has the form

$$
\left\{\begin{array}{l}
\gamma^{\prime}(x)=i\left[\kappa_{2}(x) \gamma(x)^{2}+\kappa_{1}(x) \gamma(x), \sigma_{2}\right], \quad x \in[0, l], \\
\gamma(0)=\gamma^{+}
\end{array}\right.
$$

By Lemma 3.12 from [7], in view of the diagonal form of $\sigma_{2},(2.23)$ is true for every solution of system (2.6). Taking this into account, system (2.6) takes the form

$$
\left\{\begin{aligned}
\gamma_{j k}^{\prime}(x) & =i\left(b_{j}-b_{k}\right)\left(\kappa_{1}(x)+\kappa_{2}(x)\left(\gamma_{j j}+\gamma_{k k}\right)\right) \\
& +i\left(b_{j}-b_{k}\right) \kappa_{2}(x) \sum_{\substack{s=1 \\
s \neq j, k}}^{n} \gamma_{j s}(x) \gamma_{s k}(x), \quad x \in[0, l], \quad j \neq k \\
\gamma_{k j}(x) & =\overline{\gamma_{j k}(x)}, \quad x \in[0, l], \quad j \neq k \\
\gamma_{j k}(0) & =\gamma_{j k}, \quad j \neq k
\end{aligned}\right.
$$

We search the solution of this system in the form of (2.24). In view of (2.1), $\gamma_{j j}=\alpha_{1} b_{j}+\alpha_{0}, j \in\{1, \ldots, n\}$, holds. Therefore formula (2.24) becomes

$$
\gamma_{j k}(x)=i E_{j}(x) / E_{k}(x) y_{j k}(x), \quad E_{j}(x):=\mathrm{e}^{i b_{j}\left(K_{1}(x)+\left(\alpha_{1} b_{j}+2 \alpha_{0}\right) K_{2}(x)\right)} .
$$

After substituting formula (2.8) into system (2.7), it is easy to check that it is equivalent to (2.5). 
Now let the matrix $C$ be real. We are to prove that every solution of system (2.5) is real. Let $\left\{y_{j k}(\cdot)\right\}_{j \neq k}$ be some (complex-valued) solution of system $(2.5)$. Let

$$
u_{j k}(\cdot):=\operatorname{Re} y_{j k}(\cdot), \quad v_{j k}(\cdot):=\operatorname{Im} y_{j k}(\cdot), \quad j \neq k .
$$

By separating the imaginary part from the equations of problem (2.5) and taking into account that $c_{j k} \in R, j \neq k$, we obtain the following system on the functions $v_{j k}(\cdot)$ :

$$
\left\{\begin{array}{l}
v_{j k}^{\prime}=\left(b_{k}-b_{j}\right) \kappa_{2}(x) \sum_{\substack{s=1 \\
s \neq j, k}}^{n}\left(u_{s k} v_{j s}+u_{j s} v_{s k}\right), \quad x \in[0, l], \quad j \neq k, \\
v_{j k}(0)=0, \quad j \neq k .
\end{array}\right.
$$

System (2.10) is a Cauchy problem for the system of linear ordinary differential equations with zero initial data. Therefore, by the uniqueness theorem, $v_{j k}(\cdot)=0$, $j \neq k$, which signifies the reality of the solution $\left\{y_{j k}(\cdot)\right\}_{j \neq k}$.

Theorem 2.2. Let $n=4$,

$$
\begin{gathered}
b_{4}<b_{1}<b_{2}<b_{3}, \quad b_{1}+b_{2}=b_{3}+b_{4}, \\
\alpha_{3}:=\frac{b_{3}-b_{2}}{b_{3}-b_{1}}, \quad \alpha_{4}:=\frac{b_{4}-b_{2}}{b_{4}-b_{1}} .
\end{gathered}
$$

Further, let

$$
\begin{gathered}
c_{j k} \in R, \quad c_{j k}=-c_{k j}, \quad j, k \in\{1,2,3,4\}, \\
c_{1 k}>0, \quad k \in\{2,3,4\}, \\
c_{23}=\sqrt{\alpha_{3}} \cdot c_{13}, \quad c_{24}=-\sqrt{\alpha_{4}} \cdot c_{14}, \quad c_{34}=0 .
\end{gathered}
$$

Next suppose

$$
\begin{aligned}
\beta_{3} & :=\frac{b_{2}-b_{1}}{b_{3}-b_{1}}, \quad \beta_{4}:=\frac{b_{2}-b_{1}}{b_{4}-b_{1}}, \\
\alpha & :=\frac{c_{14}}{c_{13}}, \quad \beta:=\beta_{3}+\beta_{4} \alpha^{2}, \\
F(y) & :=\int_{c_{13}}^{y} \frac{d u}{u \sqrt{c_{12}^{2}+\beta\left(u^{2}-c_{13}^{2}\right)}} \\
\rho & :=\sqrt{\left(b_{3}-b_{1}\right)\left(b_{3}-b_{2}\right)}, \\
v(x) & :=F^{-1}\left(\rho K_{2}(x)\right),
\end{aligned}
$$


where $F^{-1}(\cdot)$ is the function inverse to the function $F(\cdot)$. Let $\left(y_{0}^{-}, y_{0}^{+}\right) \subset \bar{R}$ be the largest by inclusion interval containing the number $c_{13}$, and the inequality

$$
c_{12}^{2}+\beta\left(y^{2}-c_{13}^{2}\right)>0, \quad y_{0}^{-}<y<y_{0}^{+},
$$

be true. Further, let $\kappa_{0}, \kappa_{1} \kappa_{2} \in L^{1}[0, l]$ be real functions and the functions $K_{1}(x)$, $K_{2}(x)$ be given by the equalities $K_{j}(x):=\int_{0}^{x} \kappa_{j}(t) d t, j=1,2$, and

$$
F\left(y_{0}^{-}\right)<\rho K_{2}(x)<F\left(y_{0}^{+}\right), \quad x \in[0, l) .
$$

Then the pair $\{a(\cdot), \gamma(\cdot)\}$, where $a(x)=\kappa_{2}(x) \gamma(x)^{2}+\kappa_{1}(x) \gamma(x)+\kappa_{0}(x), x \in[0, l]$, is the solution of system (1.2) if and only if the equalities

$$
\begin{aligned}
\gamma_{j j}(x) & =\gamma_{j j}^{+}, \quad j \in\{1, \ldots, n\}, \\
\gamma_{j k}(x) & =i \mathrm{e}^{i\left(b_{j}-b_{k}\right)\left(K_{1}(x)+\left(\gamma_{j j}^{+}+\gamma_{k k}^{+}\right) K_{2}(x)\right)} y_{j k}(x), \quad j \neq k,
\end{aligned}
$$

hold as $x \in[0, l]$, where the functions $y_{j k}, j \neq k$ are given by

$$
\begin{aligned}
& y_{12}(x)=\sqrt{c_{12}^{2}+\beta\left(v^{2}(x)-c_{13}^{2}\right)}, \\
& y_{13}(x)=v(x), \\
& y_{14}(x)=\alpha v(x), \\
& y_{23}(x)=\sqrt{\alpha_{3}} v(x), \\
& y_{24}(x)=-\sqrt{\alpha_{4}} \alpha v(x), \\
& y_{34}(x)=0, \\
& y_{k j}(x)=y_{j k}(x), \quad 1 \leqslant j<k \leqslant 4 .
\end{aligned}
$$

P r o o f. First check that the set $\left\{y_{12}(\cdot), y_{13}(\cdot), y_{14}(\cdot), y_{23}(\cdot), y_{24}(\cdot), y_{34}(\cdot)\right\}$ is the solution of system (2.5). Inequality (2.22) implies that the function $v(\cdot)$, given by formula (2.20), is correctly defined on the segment $[0, l]$, besides, $v(x) \in$ $\left(y_{0}^{-}, y_{0}^{+}\right), x \in[0, l)$. Therefore the equality

$$
F(v(x))=\rho K_{2}(x), \quad x \in[0, l],
$$

is true. Differentiating it, we obtain

$$
v^{\prime}(x) F^{\prime}(v(x))=\rho \kappa_{2}(x), \quad x \in[0, l] .
$$

Hence, taking into account (2.18), (2.25), (2.26), (2.28), we obtain

$$
\begin{aligned}
y_{13}^{\prime}(x) & =v^{\prime}(x)=\rho \kappa_{2}(x) v(x) \sqrt{c_{12}^{2}+\beta\left(v^{2}(x)-c_{13}^{2}\right)} \\
& =\rho \kappa_{2}(x) v(x) y_{12}(x) \\
& =\frac{\rho}{\sqrt{\alpha_{3}}} \kappa_{2}(x) y_{12}(x) y_{23}(x) \\
& =\left(b_{3}-b_{1}\right) \kappa_{2}(x) y_{12}(x) y_{23}(x), \quad x \in[0, l] .
\end{aligned}
$$


Note that accordingly to (2.11), the equality $\alpha_{3} \alpha_{4}=1$ is true. Therefore,

$$
\frac{\sqrt{\alpha_{3}}\left(b_{1}-b_{3}\right)}{\sqrt{\alpha_{4}}}=\alpha_{3}\left(b_{1}-b_{3}\right)=b_{2}-b_{3}=b_{4}-b_{1},
$$

and in virtue of $(2.26),(2.27),(2.33),(2.28), \quad(2.29)$, we have

$$
\begin{aligned}
y_{14}^{\prime}(x) & =\alpha y_{13}^{\prime}(x)=\alpha\left(b_{3}-b_{1}\right) \kappa_{2}(x) y_{12}(x) y_{23}(x) \\
& =\frac{\sqrt{\alpha_{3}}\left(b_{1}-b_{3}\right)}{\sqrt{\alpha_{4}}} \kappa_{2}(x) y_{12}(x) y_{24}(x) \\
& =\left(b_{4}-b_{1}\right) \kappa_{2}(x) y_{12}(x) y_{24}(x), \quad x \in[0, l] .
\end{aligned}
$$

Then we have

$$
\begin{aligned}
y_{23}^{\prime}(x) & =\sqrt{\alpha_{3}} y_{13}^{\prime}(x)=\sqrt{\alpha_{3}}\left(b_{3}-b_{1}\right) \kappa_{2}(x) y_{12}(x) y_{23}(x) \\
& =\alpha_{3}\left(b_{3}-b_{1}\right) \kappa_{2}(x) y_{12}(x) y_{13}(x) \\
& =\left(b_{3}-b_{2}\right) \kappa_{2}(x) y_{12}(x) y_{13}(x), \quad x \in[0, l], \\
y_{24}^{\prime}(x)= & -\sqrt{\alpha_{4}} y_{14}^{\prime}(x)=-\sqrt{\alpha_{4}}\left(b_{4}-b_{1}\right) \kappa_{2}(x) y_{12}(x) y_{24}(x) \\
= & \alpha_{4}\left(b_{4}-b_{1}\right) \kappa_{2}(x) y_{12}(x) y_{14}(x) \\
= & \left(b_{4}-b_{2}\right) \kappa_{2}(x) y_{12}(x) y_{14}(x), \quad x \in[0, l] .
\end{aligned}
$$

From (2.25) and (2.32), we obtain

$$
y_{12}^{\prime}(x)=\frac{\beta v(x) v^{\prime}(x)}{y_{12}(x)}=\rho \beta \kappa_{2}(x) v^{2}(x), \quad x \in[0, l] .
$$

Further, taking into account (2.26)-(2.29), we have

$$
y_{13}(x) y_{23}(x)+y_{14}(x) y_{24}(x)=\left(\sqrt{\alpha_{3}}-\sqrt{\alpha_{4}} \alpha^{2}\right) v^{2}(x), \quad x \in[0, l] .
$$

Note that in virtue of (2.11) the equality

$$
\rho=\sqrt{\left(b_{3}-b_{2}\right)\left(b_{3}-b_{1}\right)}=\sqrt{\left(b_{4}-b_{2}\right)\left(b_{4}-b_{1}\right)}
$$

is true. Therefore, since $b_{4}<b_{1}<b_{3}$, then

$$
\begin{aligned}
\rho \beta & =\rho\left(\beta_{3}+\beta_{4} \alpha^{2}\right)=\sqrt{\left(b_{3}-b_{2}\right)\left(b_{3}-b_{1}\right)} \frac{b_{2}-b_{1}}{b_{3}-b_{1}} \\
& +\sqrt{\left(b_{4}-b_{2}\right)\left(b_{4}-b_{1}\right)} \frac{b_{2}-b_{1}}{b_{4}-b_{1}} \alpha^{2} \\
& =\left(b_{2}-b_{1}\right)\left(\sqrt{\frac{b_{3}-b_{2}}{b_{3}-b_{1}}}-\sqrt{\frac{b_{4}-b_{2}}{b_{4}-b_{1}}} \alpha^{2}\right) \\
& =\left(b_{2}-b_{1}\right)\left(\sqrt{\alpha_{3}}-\sqrt{\alpha_{4}} \alpha^{2}\right) .
\end{aligned}
$$


Now the relations (2.37)-(2.40) yield

$$
y_{12}^{\prime}(x)=\left(b_{2}-b_{1}\right) \kappa_{2}(x)\left(y_{13}(x) y_{23}(x)+y_{14}(x) y_{24}(x)\right), x \in[0, l] .
$$

Since $\alpha_{3} \alpha_{4}=1$, then in virtue of $(2.26)-(2.30)$,

$$
\begin{aligned}
& \left(b_{4}-b_{3}\right) \kappa_{2}(x)\left(y_{13}(x) y_{14}(x)+y_{23}(x) y_{24}(x)\right) \\
& =\left(b_{4}-b_{3}\right) \kappa_{2}(x)\left(y_{13}(x) y_{14}(x)-\sqrt{\alpha_{3}} y_{13}(x) \sqrt{\alpha_{4}} y_{14}(x)\right) \\
& =0=y_{34}^{\prime}(x)
\end{aligned}
$$

Now the equalities $(2.41),(2.33),(2.34),(2.35),(2.36),(2.42)$ imply that the functions $y_{12}, y_{13}, y_{14}, y_{23}, y_{24}, y_{34}$ satisfy the equations of system (2.5). Further, since $c_{12}>0$, then

$$
\begin{aligned}
& y_{13}(0)=v(0)=F^{-1}\left(\rho K_{2}(0)\right)=F^{-1}(0)=c_{13}, \\
& y_{12}(0)=\sqrt{c_{12}^{2}+\beta\left(v^{2}(0)-c_{13}^{2}\right)}=c_{12}, \\
& y_{14}(0)=\alpha v(0)=\frac{c_{14}}{c_{13}} c_{13}=c_{14} \\
& y_{23}(0)=\sqrt{\alpha_{3}} y_{13}(0)=\sqrt{\alpha_{3}} c_{13}=c_{23} \\
& y_{24}(0)=-\sqrt{\alpha_{4}} y_{14}(0)=-\sqrt{\alpha_{4}} c_{14}=c_{24} \\
& y_{34}(0)=0=c_{34}
\end{aligned}
$$

Thus the functions $y_{12}(\cdot), y_{13}(\cdot), y_{14}(\cdot), y_{23}(\cdot), y_{24}(\cdot), y_{34}(\cdot)$ satisfy also the initial data of system (2.5). General uniqueness theorems for the initial problem imply that this system has the unique solution on $[0, l]$.

We will show the way of finding expressions for $y_{k s}(\cdot), k, s \in 1, \ldots, n$ (2.25) - (2.31) as solutions of the corresponding system of differential equations (2.5). Let now the functions $y_{12}(\cdot), y_{13}(\cdot), y_{14}(\cdot) y_{23}(\cdot), y_{24}(\cdot), y_{34}(\cdot) \in A C[0, l]$ satisfy problem (2.5). Multiply equation $(2.33)$ by $\left(b_{3}-b_{2}\right) y_{13}(x)$ and equation $(2.35)$ by $\left(b_{3}-b_{1}\right) y_{23}(x)$. After summarizing the results, we arrive at the relation $y_{23}^{\prime}(x) y_{23}(x)-\alpha_{3} y_{13}^{\prime}(x) y_{13}(x)=0$, integrating which we obtain

$$
y_{23}(x)=\sqrt{c_{23}^{2}+\alpha_{3}\left(y_{13}^{2}(x)-c_{13}^{2}\right)} .
$$

Taking into account (2.15), we have (2.28). Following the same procedure with (2.34) and (2.36), we get $y_{24}(x)=\sqrt{c_{24}^{2}+\alpha_{4}\left(y_{14}^{2}(x)-c_{14}^{2}\right)}$. After using (2.15), we obtain

$$
y_{24}(x)=-\sqrt{\alpha_{4}} y_{14}(x) .
$$

Use (2.49) and (2.50) for the equations (2.33) and (2.34) to get

$$
\begin{gathered}
y_{13}^{\prime}(x)=\kappa_{2}(x)\left(b_{3}-b_{1}\right) y_{12}(x) \sqrt{\alpha_{3}} y_{13}(x), \\
y_{14}^{\prime}(x)=\kappa_{2}(x)\left(b_{4}-b_{1}\right) y_{12}(x) \sqrt{\alpha_{4}} y_{14}(x) .
\end{gathered}
$$


Express $\kappa_{2}(x) y_{12}(x)$ from (2.51) and taking into account (2.39) equate the obtained relations

$$
\frac{y_{13}^{\prime}(x)}{y_{13}(x)}=\frac{y_{14}^{\prime}(x)}{y_{14}(x)}
$$

Thus (2.53) yields

$$
y_{14}(x)=\frac{c_{14}}{c_{13}} y_{13}(x)=\alpha y_{13}(x) .
$$

Thus the correctness of equality (2.27) is confirmed. After substituting it into (2.50), the correctness of (2.29) is also confirmed. By using the obtained results, we transform the equations (2.41) and (2.33), subject to (2.40),

$$
\begin{aligned}
y_{12}^{\prime}(x) & =\kappa_{2}(x)\left(b_{2}-b_{1}\right) y_{13}^{2}(x)\left(\sqrt{\alpha_{3}}-\alpha^{2} \sqrt{\alpha_{4}}\right) \\
& =\kappa_{2}(x) \rho \beta y_{13}^{2}(x) ; \\
y_{13}^{\prime}(x) & =\kappa_{2}(x) \rho y_{12}(x) y_{13} .
\end{aligned}
$$

Whence we obtain that

$$
\frac{y_{12}^{\prime}(x)}{y_{13}(x) \beta}=\frac{y_{13}^{\prime}(x)}{y_{12}(x)}
$$

or

$$
y_{12}(x)=\sqrt{c_{12}^{2}+\beta\left(y_{13}^{2}(x)-c_{13}^{2}\right)} .
$$

Thus (2.25) takes place. Substituting the obtained expressions for $y_{12}(x)(2.25)$ and $y_{23}(x)(2.28)$ into the equation for $y_{13}^{\prime}(x)(2.33)$, we obtain

$$
y_{13}^{\prime}(x)=\kappa_{2}(x) \rho y_{13}(x) \sqrt{c_{12}^{2}+\beta\left(y_{13}^{2}(x)-c_{13}^{2}\right)},
$$

or, using the notation (2.19),

$$
\frac{y_{13}^{\prime}(x)}{y_{13}(x) \sqrt{c_{12}^{2}+\beta\left(y_{13}^{2}(x)-c_{13}^{2}\right)}}=\rho \kappa_{2}(x)
$$

It is easy to see that (2.60), subject to notation (2.18), becomes (2.26).

Corollary 2.3. In the conditions of Theorem 2 suppose $c_{12}=0$ which determines the form of the matrix $C$ in (2.1) in the following way:

$$
i C=\left(\begin{array}{cccc}
0 & 0 & c_{13} & c_{14} \\
0 & 0 & c_{23} & c_{24} \\
\overline{c_{13}} & \overline{c_{23}} & 0 & 0 \\
\overline{c_{14}} & \overline{c_{24}} & 0 & 0
\end{array}\right)
$$


Then for the functions $y_{12}(\cdot), y_{13}(\cdot)$ the following representations take place:

$$
\begin{aligned}
& y_{12}(x)=c_{13} \sqrt{\beta} \tan (z(x)), \beta>0, \\
& y_{13}(x)=\frac{c_{13}}{\cos (z(x))}, \beta>0,
\end{aligned}
$$

or

$$
\begin{aligned}
& y_{12}(x)=c_{13} \sqrt{\beta}, \beta<0 \\
& y_{13}(x)=0
\end{aligned}
$$

or

$$
\begin{aligned}
& y_{12}(x)=c_{13} \sqrt{\beta} \operatorname{th}(z(x)), \beta<0 \\
& y_{13}(x)=\frac{c_{13}}{\operatorname{ch}(z(x))}, \beta<0
\end{aligned}
$$

where

$$
z(x)=c_{13} \rho \sqrt{\beta} K_{2}(x)
$$

P r o o f. Consider the case $\beta>0$. The definition of the function $F(\cdot)$ and formula (2.26) imply that

$$
\rho K_{2}(x)=F\left(y_{13}(x)\right)=\frac{1}{\sqrt{\beta}} \int_{c_{13}}^{y_{13}(x)} \frac{d u}{u \sqrt{u^{2}-c_{13}^{2}}} .
$$

Then (2.68) has the form

$$
-c_{13} \sqrt{\beta} r h o K_{2}(x)=\arcsin \left|\frac{c_{13}}{y_{13}(x)}\right|-\frac{\pi}{2} .
$$

Using notation (2.67), we obtain

$$
\begin{aligned}
-\sin (z(x)) & =\sin \left(\arcsin \left|\frac{c_{13}}{y_{13}(x)}\right|-\frac{\pi}{2}\right) \\
= & \sin \left(\arcsin \left|\frac{c_{13}}{y_{13}(x)}\right|\right) \cos \frac{\pi}{2}-\cos \left(\arcsin \left|\frac{c_{13}}{y_{13}(x)}\right|\right) \sin \frac{\pi}{2} \\
& =-\sqrt{1-\frac{c_{13}^{2}}{y_{13}^{2}(x)}} .
\end{aligned}
$$


Suppose $z(x)$ is such that $\sin (z(x))$ and $\cos (z(x))$ are positive, then we obtain (2.62). Substituting (2.62) into (2.25)-(2.30), we will come to the form of $(2.61)$ for $y_{12}(\cdot)$ and to the corresponding expressions for the functions $y_{14}(\cdot)$, $y_{23}(\cdot), y_{24}(\cdot), y_{34}(\cdot)$. Thus,

In the case $\beta<0$, in virtue of inequality (2.21), we obtain that $y_{13}^{2}(x)-c_{13}^{2}<0$.

$$
\rho K_{2}(x)=F\left(y_{13}(x)\right)=\frac{1}{\sqrt{\beta}} \int_{c_{13}}^{y_{13}(x)} \frac{d u}{u \sqrt{c_{13}^{2}-u^{2}}} .
$$

Whence it follows that

$$
-z(x)=\ln \left(\frac{\sqrt{c_{13}^{2}-u^{2}}+c_{13}}{y_{13}(x)}\right)
$$

or

$$
\sqrt{c_{13}^{2}-y_{13}^{2}(x)}=y_{13}(x) \exp ^{-z(x)}-c_{13}
$$

As a result of transformations, we have

$$
y_{13}(x)\left(y_{13}(x)\left(\exp ^{-2 z(x)}+1\right)-2 \exp ^{-z(x)} c_{13}\right)=0,
$$

i.e., either $y_{13}(x)=0$, which results in the solutions of $(2.63)-(2.64)$, or $y_{13}(x)=$ $\frac{2 c_{13}}{\exp ^{z(x)}+\exp ^{-z(x)}}$, which corresponds to the solutions in the forms of $(2.65)$ and (2.66).

Ex a m p l e 2.4. Let

$$
\gamma^{+}=\left(\begin{array}{cccc}
0 & 0 & 2 i & i \\
0 & 0 & \sqrt{2} i & -\sqrt{2} i \\
-2 i & -\sqrt{2} i & 0 & 0 \\
-i & \sqrt{2} i & 0 & 0
\end{array}\right), \quad \sigma_{2}=\left(\begin{array}{cccc}
0 & 0 & 0 & 0 \\
0 & b & 0 & 0 \\
0 & 0 & 2 b & 0 \\
0 & 0 & 0 & -b
\end{array}\right), \quad b>0
$$

Then the pair $\{a(x), \gamma(x)\}$, where $a(x)=\gamma^{2}(x)$, is the solution of system (1.2) if and only if

$$
\gamma(x)=\left(\begin{array}{cccc}
0 & \sqrt{2} \operatorname{tg}(2 b x) & \frac{2}{\cos (2 b x)} & \frac{1}{\cos (2 b x)} \\
-\sqrt{2} \operatorname{tg}(2 b x) & 0 & \frac{\sqrt{2}}{\cos (2 b x)} & -\frac{\sqrt{2}}{\cos (2 b x)} \\
-\frac{2}{\cos (2 b x)} & -\frac{\sqrt{2}}{\cos (2 b x)} & 0 & 0 \\
-\frac{1}{\cos (2 b x)} & \frac{\sqrt{2}}{\cos (2 b x)} & 0 & 0
\end{array}\right) .
$$




\section{Case of Cubic Dependency of $a(x)$ from $\gamma(x)$}

Remind the statement proved in [7].

Theorem 3.1. Let

$$
\gamma^{+}=\operatorname{diag}\left(l_{1} I_{n_{1}}, \ldots, l_{r} I_{n_{r}}\right), \quad n_{1}+\ldots n_{r}=n,
$$

where $l_{1}, \ldots, l_{r}$ are different real numbers.

Then for every solution $\{a(\cdot), \gamma(\cdot)\}$ of system (1.2) there exists a unique unitary matrix function $U \in A C\left([0, l] ; C^{n \times n}\right)$ such that

$$
\gamma(x)=U(x) \gamma^{+} U^{*}(x), \quad x \in[0, l], \quad U(0)=I_{n},
$$

and the matrix function $C(\cdot):=-i U^{-1}(\cdot) U^{\prime}(\cdot)$ is self-adjoint and has the zero block diagonal relative to the decomposition $C^{n}=C^{n_{1}} \oplus \ldots \oplus C^{n_{r}}$. Besides,

$$
a(x)=U(x) A(x) U^{*}(x), \quad x \in[0, l],
$$

where

$$
\begin{gathered}
A(\cdot)=\operatorname{diag}\left(A_{1}(\cdot), \ldots, A_{r}(\cdot)\right)=A^{*}(\cdot), \\
A_{j} \in L^{1}\left([0, l] ; C^{n_{j} \times n_{j}}\right), \quad j \in\{1, \ldots, r\} .
\end{gathered}
$$

Moreover, for $B(x):=U^{*}(x) \sigma_{2} U(x)$,

$$
\begin{aligned}
{\left[C(x), \gamma^{+}\right] } & =[A(x), B(x)], \quad x \in[0, l], \\
B^{\prime}(x) & =i[B(x), C(x)], \quad x \in[0, l], \quad B(0)=\sigma_{2}
\end{aligned}
$$

take place.

Conversely, if for the self-adjoint matrix functions

$$
A, C \in L^{1}\left([0, l] ; C^{n \times n}\right), \quad B \in A C\left([0, l] ; C^{n \times n}\right),
$$

(3.4), (3.5), (3.6), (3.7) take place, and $U \in A C\left([0, l] ; C^{n \times n}\right)$ is the solution of the initial problem

$$
U^{\prime}(x)=i U(x) C(x), \quad x \in[0, l], \quad U(0)=I_{n},
$$

then $U(x)$ is unitary for every $x \in[0, l]$,

$$
B(x)=U^{*}(x) \sigma_{2} U(x), \quad x \in[0, l],
$$

and the pair $\{a(\cdot), \gamma(\cdot)\}$, given by (3.2), (3.3), is the solution of system (1.2).

Theorem provides the following step-by-step procedure [7] for finding all the solutions of system (1.2):

1. Choose an orthonormal basis in $C^{n}$, in which the matrix $\gamma^{+}$has the diagonal form (3.1). 
2. Choose an arbitrary matrix function $A(\cdot)$ satisfying conditions (3.4)-(3.5).

3. Solve the Cauchy problem for the nonlinear system of ordinary differential equations on the matrix $B$ obtained from (3.6), (3.7) in the previous remark.

4. If it has the global solution on the segment $[0, l]$, then we calculate the matrix $C(x)$ by the formula

$$
\begin{aligned}
& C_{j k}(x)=\left(l_{k}-l_{j}\right)^{-1}\left(A_{j}(x) B_{j k}(x)-B_{j k}(x) A_{k}(x)\right), \\
& \quad x \in[0, l], \quad j, k \in\{1, \ldots, r\}, \quad j \neq k,
\end{aligned}
$$

supposing that its diagonal blocks are equal to zero.

5. Find $U(\cdot)$ as a unique solution of the Cauchy problem (3.9) for the system of linear ordinary differential equations.

6. Finally, we obtain the solution $\{a(\cdot), \gamma(\cdot)\}$ of system (1.2) by the formulas (3.2), (3.3).

Using Theorem 3.1, we will show how to find the explicit form of the matrix $B(x)$ for the case $n=4$ and $a(x)=\kappa(x) \gamma^{3}(x)$, where $\kappa(x)$ is a real function such that $\kappa \in L^{1}[0, l]$.

Theorem 3.2. Let $n=4, \lambda_{1}, \ldots, \lambda_{4}$ be different real numbers such that $\lambda_{3}$ and $\lambda_{4}$ are between $\lambda_{1}$ and $\lambda_{2}$,

$$
\begin{aligned}
\gamma^{+} & =\operatorname{diag}\left(\lambda_{1}, \ldots, \lambda_{4}\right), \\
\sigma_{2} & =\left(b_{j k}^{(0)}\right)_{j, k=1}^{4}=i \beta_{j k}, \beta_{j k}>0, \beta_{j k} \in R \text { as } j \neq k, \\
b_{j j}^{(0)} & =\beta_{1} \lambda_{j}+\beta_{0}, j \in\{\overline{1,4}\}, \\
C(x) & =\left(c_{j k}(x)\right)_{j, k=1}^{4}, \text { besides } \quad c_{j j}=0, \\
a(x) & =\kappa(x) \gamma^{3}(x),
\end{aligned}
$$

where $a(x)$ is a matrix function and $\kappa(x) \in L^{1}[0, l]$ is a real function, $x \in[0, l]$, and the matrix $B$ is such that $B(x)=\left(b_{j k}(x)\right)_{j, k=1}^{4}=B^{*}(x)$ as $j \neq k$.

Let further

$$
\begin{gathered}
\alpha_{j}=\sqrt{\frac{\lambda_{2}-\lambda_{j}}{\lambda_{j}-\lambda_{1}}}, \beta_{j}=\frac{\lambda_{2}-\lambda_{1}}{\lambda_{j}-\lambda_{1}}, j=3,4, \\
\alpha=\frac{\sqrt{\left(\lambda_{4}-\lambda_{1}\right)\left(\lambda_{2}-\lambda_{4}\right)}\left(\lambda_{1}+\lambda_{2}+\lambda_{4}\right)}{\sqrt{\left(\lambda_{3}-\lambda_{1}\right)\left(\lambda_{2}-\lambda_{3}\right)}\left(\lambda_{1}+\lambda_{2}+\lambda_{3}\right)},
\end{gathered}
$$




$$
\begin{gathered}
\psi(y)=\sqrt{\beta_{12}^{2}-\beta_{3}\left(y^{2}-\beta_{13}^{2}\right)-\beta_{4} \beta_{14}^{2}\left[\left(\frac{y}{\beta_{13}}\right)^{2 \alpha}-1\right]}, \\
F(y)=\int_{\beta_{13}}^{y} \frac{d t}{t \psi(t)} .
\end{gathered}
$$

Then the elements of the matrix $B(x)$ from (3.10) are given by

$$
b_{j k}(x)=i \exp ^{-i \beta_{1} \cdot\left(\lambda_{j}^{3}-\lambda_{k}^{3}\right) \int_{0}^{x} \kappa(t) d t} y_{j k}(x), j \neq k,
$$

where the real functions $y_{j k}(\cdot)(j \neq k)$ are given by the equalities

$$
\begin{aligned}
y_{12}(x) & =\psi\left(y_{13}(x)\right), \\
y_{13}(x) & =F^{-1}\left(\sqrt{\left(\lambda_{3}-\lambda_{1}\right)\left(\lambda_{2}-\lambda_{3}\right)} \int_{0}^{x} \kappa(t) d t\right), \\
y_{14}(x) & =\beta_{14}\left(\frac{y_{13}(x)}{\beta_{13}}\right)^{\alpha}, \\
y_{2 j}(x) & =\alpha_{j} y_{1 j}(x), \quad(j=3,4), \\
y_{34}(x) & =0 \\
y_{k j}(\cdot) & =y_{j k}(\cdot), \quad(j \neq k) .
\end{aligned}
$$

Here $F^{-1}(\cdot)$ is a function inverse to the monotonously increasing function $F(\cdot)$.

P r o o f. (3.6) yields the relation

$$
c_{j k}(x)=-\kappa(x) d_{j k} b_{j k}(x),
$$

where $d_{j k}=\frac{\lambda_{j}^{3}-\lambda_{k}^{3}}{\lambda_{j}-\lambda_{k}}=\lambda_{j}^{2}+\lambda_{j} \lambda_{k}+\lambda_{k}^{2}$. Substitute (3.23) into (3.7), then

$$
\begin{aligned}
& b_{j k}^{\prime}(x)=-i \kappa(x) d_{j k}\left(b_{j j}^{(0)}-b_{k k}^{(0)}\right) b_{j k}(x)- \\
& i \kappa(x) \sum_{l=1, l \neq k}^{4}\left(d_{k l}-d_{j l}\right) b_{j l}(x) b_{l k}(x),
\end{aligned}
$$

where $j \neq k$, and $d_{j k}\left(b_{j j}^{(0)}-b_{k k}^{(0)}\right)=\beta_{1}\left(\lambda_{j}^{3}-\lambda_{k}^{3}\right)$. It is easy to see that

$$
d_{k l}-d_{j l}=\lambda_{k}^{2}+\lambda_{k} \lambda_{l}+\lambda_{l}^{2}-\lambda_{j}^{2}-\lambda_{j} \lambda_{l}-\lambda_{l}^{2}=\left(\lambda_{k}-\lambda_{j}\right)\left(\lambda_{j}+\lambda_{k}+\lambda_{l}\right) .
$$


Let $b_{j k}(x)$ be given by (3.16). Then

$$
\left\{\begin{array}{l}
y_{j k}^{\prime}(x)=\kappa(x) \sum_{l=1, l \neq j, k}^{4}\left(d_{k l}-d_{j l}\right) y_{j l}(x) y_{l k}(x), \\
y_{j k}(0)=\beta_{j k} .
\end{array}\right.
$$

Use the fact that $y_{j k}(x)$ are real and $y_{34}(x)=0$. Then $\beta_{34}=0, y_{j k}(x)=y_{k j}(x)$, as $1 \leq j<k \leq 4$, and the system has the form

$$
\begin{aligned}
& y_{12}^{\prime}=\kappa(x)\left(d_{13}-d_{23}\right) y_{13} y_{23}+\kappa(x)\left(d_{14}-d_{24}\right) y_{14} y_{24}, \\
& y_{13}^{\prime}=\kappa(x)\left(d_{23}-d_{12}\right) y_{12} y_{23} \\
& y_{23}^{\prime}=\kappa(x)\left(d_{12}-d_{13}\right) y_{12} y_{13} \\
& y_{14}^{\prime}=\kappa(x)\left(d_{24}-d_{12}\right) y_{12} y_{24} \\
& y_{24}^{\prime}=\kappa(x)\left(d_{12}-d_{14}\right) y_{12} y_{14} .
\end{aligned}
$$

The condition $y_{34}(x)=0$ implies

$$
0=\kappa(x)\left(d_{13}-d_{14}\right) y_{13} y_{14}+\left(d_{23}-d_{24}\right) y_{23} y_{24} .
$$

It follows from (3.27) and (3.28) that

$$
\left(d_{23}-d_{12}\right)\left(y_{23}^{2}(x)-\beta_{23}^{2}\right)=\left(d_{12}-d_{13}\right)\left(y_{13}^{2}(x)-\beta_{13}^{2}\right)
$$

or

$$
\left(\lambda_{3}-\lambda_{1}\right)\left(y_{23}^{2}(x)-\beta_{23}^{2}\right)=\left(\lambda_{2}-\lambda_{3}\right)\left(y_{13}^{2}(x)-\beta_{13}^{2}\right) .
$$

The restriction on $\lambda_{j}$, where $j=\overline{1,4}$, follows from the relation

$$
\left(\lambda_{3}-\lambda_{1}\right) \beta_{23}^{2}=\left(\lambda_{2}-\lambda_{3}\right) \beta_{13}^{2},
$$

i.e., $\left(\lambda_{3}-\lambda_{1}\right)\left(\lambda_{2}-\lambda_{3}\right)>0$, thus $\lambda_{3}$ is between $\lambda_{1}$ and $\lambda_{2}$. Therefore,

$$
y_{23}(x)=\sqrt{\frac{\lambda_{2}-\lambda_{3}}{\lambda_{3}-\lambda_{1}}} y_{13}(x)
$$

Analogously, (3.29) and (3.30) imply that

$$
y_{24}(x)=\sqrt{\frac{\lambda_{2}-\lambda_{4}}{\lambda_{4}-\lambda_{1}}} y_{14}(x)
$$

if $\left(\lambda_{4}-\lambda_{1}\right) \beta_{24}^{2}=\left(\lambda_{2}-\lambda_{4}\right) \beta_{14}^{2}$, and thus $\lambda_{4}$ is between $\lambda_{1}$ and $\lambda_{2}$. Taking into account (3.12), we write equality (3.31) in the form

$$
\left(\lambda_{1}+\lambda_{3}+\lambda_{4}+\alpha_{3} \alpha_{4}\left(\lambda_{2}+\lambda_{3}+\lambda_{4}\right)\right) y_{13}(x) y_{14}(x)=0
$$


besides, $\beta_{23}=\alpha_{3} \beta_{13}, \beta_{24}=\alpha_{4} \beta_{14}$. Thus $y_{2 j}(x)=\alpha_{j} y_{1 j}(x)$ for $j=3$, 4. From (3.26), (3.27), (3.29), we find

$$
y_{12}^{2}(x)-\beta_{12}^{2}=-\beta_{3}\left(y_{13}^{2}(x)-\beta_{13}^{2}\right)-\beta_{4}\left(y_{14}^{2}(x)-\beta_{14}^{2}\right) .
$$

So the equations (3.27) and (3.29) become

$$
\begin{aligned}
& y_{13}^{\prime}=\kappa(x)\left(d_{23}-d_{12}\right) \alpha_{3} y_{12} y_{13} ; \\
& y_{14}^{\prime}=\kappa(x)\left(d_{24}-d_{12}\right) \alpha_{4} y_{12} y_{14} .
\end{aligned}
$$

Since $\frac{y_{14}^{\prime}}{y_{14}}=\frac{\alpha_{4}}{\alpha_{3}} \frac{\left(d_{24}-d_{12}\right)}{\left(d_{23}-d_{12}\right)} \frac{y_{13}^{\prime}}{y_{13}}$, then denoting $\alpha=\frac{\left(d_{24}-d_{12}\right) \alpha_{4}}{\left(d_{23}-d_{12}\right) \alpha_{3}}$ (which is equivalent to (3.13)), we search $y_{14}(x)$ (subject to the initial conditions) in the form $y_{14}(x)=\beta_{14}\left(\frac{y_{13}(x)}{\beta_{13}}\right)^{\alpha}$. Substitute

$$
y_{12}(x)=\psi\left(y_{13}(x)\right)
$$

into (3.38), where

$$
\begin{aligned}
& \psi\left(y_{13}(x)\right)= \\
& \sqrt{\beta_{12}^{2}-\beta_{3}\left(y_{13}^{2}(x)-\beta_{13}^{2}\right)-\beta_{4} \beta_{14}^{2}\left(\left(\frac{y_{13}(x)}{\beta_{13}}\right)^{2 \alpha}-1\right)} .
\end{aligned}
$$

By using (3.39), we can find

$$
y_{13}^{\prime}(x)=\kappa(x) \psi\left(y_{13}(x)\right) y_{13}(x) .
$$

Since $F(y)$ is represented in the form (3.15), then

$$
y_{13}(x)=F^{-1}\left(\sqrt{\left(\lambda_{3}-\lambda_{1}\right)\left(\lambda_{2}-\lambda_{3}\right)} \int_{0}^{x} \kappa(t) d t\right) .
$$

Thus, in the case $n=4$ and cubic dependency of $a(x)$ from $\gamma(x)$, the elements of the matrix $B(x)$ are expressed in terms of elliptic functions.

Corollary 3.3. In the conditions of Theorem 3 we suppose $\lambda_{1}=-a, \lambda_{2}=a$, $\lambda_{3}=-b, \lambda_{4}=b$, where $a, b \in R$ and the condition $0<a<3 b$ is fulfilled, moreover, $\beta_{13}^{2}=\frac{1}{2}, \beta_{14}^{2}=2, \beta_{12}^{2}=\frac{3 a b-a^{2}}{a^{2}-b^{2}}$, then the solutions $y_{j k}(x)$ as $j \neq k$, 
(3.17)-(3.20), are given by

$$
\begin{aligned}
& y_{12}(x)=-i \sqrt{\frac{2 a}{a+b}} \frac{c n(z(x)+N, k) d n(z(x)+N, k)}{\operatorname{sn}(z(x)+N)} \\
& y_{13}(x)=\operatorname{sn}(z(x)+N, k) \\
& y_{14}(x)=\frac{1}{\operatorname{sn}(z(x)+N, k)} \\
& y_{23}(x)=k \operatorname{sn}(z(x)+N, k) \\
& y_{24}(x)=\frac{1}{k \operatorname{sn}(z(x)+N, k)}
\end{aligned}
$$

where

$$
\begin{aligned}
k^{2} & =\frac{a+b}{a-b}, \quad z(x)=-i \sqrt{2 a(a-b)} \int_{0}^{x} \kappa(t) d t, \\
N & =\int_{0}^{\frac{1}{\sqrt{2}}} \frac{d t}{\sqrt{\left(1-t^{2}\right)\left(1-k^{2} t^{2}\right)}} .
\end{aligned}
$$

P r o o f. From (3.18), we obtain

$$
\sqrt{(a-b)(a+b)} \int_{0}^{x} \kappa(t) d t=F\left(y_{13}(x)\right) .
$$

Applying (3.14), (3.15) and taking into account that under the given choice of $\lambda_{j}$, where $j=\overline{1,4}$, the value of $\alpha=-1$ (which is evident from (3.13)), we have

$$
\begin{gathered}
F\left(y_{13}(x)\right)=\int_{\beta_{13}}^{y_{13}(x)} \frac{d t}{\sqrt{\frac{2 a}{b-a} t^{4}+\frac{4 a^{2}}{a^{2}-b^{2}} t^{2}-\frac{2 a}{b+a}}}= \\
i \sqrt{\frac{a+b}{2 a}} \int_{\frac{1}{\sqrt{2}}}^{y_{13}(x)} \frac{d t}{\sqrt{\frac{a+b}{a-b} t^{4}+\frac{2 a}{a-b} t^{2}+1}}= \\
i \sqrt{\frac{a+b}{2 a}} \int_{\frac{1}{\sqrt{2}}}^{y_{13}(x)} \frac{d t}{\sqrt{\left(1-t^{2}\right)\left(1-\frac{a+b}{a-b} t^{2}\right)}} .
\end{gathered}
$$


Now relation (3.50) can be expressed as

$$
-i \sqrt{2 a(a-b)} \int_{0}^{x} \kappa(t) d t=\int_{\frac{1}{\sqrt{2}}}^{y_{13}(x)} \frac{d t}{\sqrt{\left(1-t^{2}\right)\left(1-\frac{a+b}{a-b} t^{2}\right)}},
$$

i.e., taking into account (3.48),

$$
z(x)=\int_{\frac{1}{\sqrt{2}}}^{y_{13}(x)} \frac{d t}{\sqrt{\left(1-t^{2}\right)\left(1-k^{2} t^{2}\right)}}
$$

or

$$
z(x)+\int_{0}^{\frac{1}{\sqrt{2}}} \frac{d t}{\sqrt{\left(1-t^{2}\right)\left(1-k^{2} t^{2}\right)}}=\int_{0}^{y_{13}(x)} \frac{d t}{\sqrt{\left(1-t^{2}\right)\left(1-k^{2} t^{2}\right)}} .
$$

In view of the notation and definition of elliptic functions, we have

$$
z(x)+N=\int_{0}^{y_{13}(x)} \frac{d t}{\sqrt{\left(1-t^{2}\right)\left(1-k^{2} t^{2}\right)}}
$$

namely,

$$
y_{13}(x)=\operatorname{sn}(z(x)+N, k) .
$$

From (3.17) and (3.14), we obtain

$$
\begin{array}{r}
y_{12}(x)=\frac{1}{s n(z(x)+N)}\left(-i \sqrt{\frac{2 a}{a+b}} \sqrt{\left(1-s n^{2}(z(x)+N)\left(1-k^{2} s n^{2}(z(x)+N)\right)\right.}\right) \\
=-i \sqrt{\frac{2 a}{a+b}} \frac{c n(z(x)+N, k) d n(z(x)+N, k)}{\operatorname{sn}(z(x)+N)} .
\end{array}
$$

Substituting the values of $\lambda_{j}$, as $j=\overline{1,4}$, we have that $\alpha_{3}=k$, and $\alpha_{4}=\frac{1}{k}$, and then (3.20) implies that $y_{23}(x)=\alpha_{3} y_{13}(x)=k \operatorname{sn}(z(x)+N, k)$ and $y_{24}(x)=$ $\alpha_{4} y_{14}(x)=\frac{1}{k \operatorname{sn}(z(x)+N, k)}$. 


\section{References}

[1] V.Ye. Zaharov, S.V. Manakov, S.P. Novikov, and L.P. Pitayevsky, Soliton Theory. Nauka, Moscow, 1980. (Russian)

[2] V.A. Zolotarev, Spectral Analisys of Non-selfadjoint Commutative Operator Systems and Nonlinear Differential Equations. - Teor. Funktsij, Funkts. Analiz, i ih pril. Kharkov, Resp. sb., 40 (1983), 68-71. (Russian)

[3] V.A. Zolotarev, Time Cones and Functional Model on Riemann Surface. - Mat. Sb. 181 (1990), No. 7, 965-994. (Russian)

[4] V.A. Zolotarev, Analitical Methods of Spectral Representations of Non-selfadjoint and Non-unitary Operators. KhNU, Kharkov, 2003. (Russian)

[5] P.D. Lax, Integrals of Nonlinear Equations of Evolution and Solitary Waves. Commun. Pure Appl. Math. 21 (1968), 467-490.

[6] M.S. Livšic and A.A. Yantsevich, Operator Colligations in Hilbert Spaces. Winston, Washington, D. C. (distributed by Wiley, New York), 1979.

[7] A.A. Lunyov and E.V. Oliynyk, On One Class of System of Lax-type Equations. UMV 10 (2013), No. 4, 507-531. (Russian) 